\title{
Stage II Childhood Hepatocellular Carcinoma AJCC v6 and v7
}

National Cancer Institute

\section{Source}

National Cancer Institute. Stage // Childhood Hepatocellular Carcinoma AJCC v6 and v7. NCl Thesaurus. Code C5709.

Stage II includes: T2, N0, M0. T2: Solitary tumor with vascular invasion or multiple tumors none more than $5 \mathrm{~cm}$. NO: No regional lymph node metastasis. M0: No distant metastasis. (AJCC 6th and 7th eds.) 